\title{
The spiral code of prime numbers
}

\author{
Vinoo Cameron \\ Hope Research, Athens, Wisconsin, USA \\ E-mail: Hope9900@frontier.com
}

\begin{abstract}
Notice: The Final Frontier in Mathematics is the spiral code of the placement of prime numbers around a half-line, as most positively the reference of empty space is a curved and cone-metric at 1:3. This spiral code is presented in brief form and the end of this manuscript. The author has nearly finished the mathematics of the polarity of the divergence/convergence at the half line of prime number values described for the first time in history by this author in his recent publications at this journal. Thus the spiral calculus will be unraveled in time and all prime number placements identified and predicted around a mathematical half-line that is critical to defining space and mass. This will allow much precision in the sciences of curved empty space and its relationship to mass, since the inverse is at a point.
\end{abstract}

The stated values at 30 constant for the half line values are as follows, although +60 is also used as a coordinate to code numbers.

The, following are the divergence codes for the half-line numbers at1:3, (10*3). SIMPLE BASIC MATHEMATICS!!! . The "sui generis" for prime number variability at 1:3 divergences is based on the following line values at 10*3 Constant. The brief file is included under the section of spiral codes

$12=+6$

$14=+2$

$16=-2$

$18=-6$

$20=-10$

$22=-14$

$24=-18$

$28=-26$

$30=-30$

$32=-5$

$34=-7$

$36=-42$

The, value at 100 half value is -170 .

The readers will not be offered the final work on the spiral calculus, which is the so called "mathematical writing on the wall", a no brainer. The author will complete that on his own time, and current mathematicians are invited to finish it.

The author first confirms the divergence/ convergence of 19 here at exact 1:3 and 1:6 as per well published manuscripts. Secondly the author presents a continuous prime number sieve at proportion 6, which is mathematically distinct from the sieve of Eratosthenes, first in history, tested by 10 million sieved numbers. Thirdly the author presents the half-line value of each and every prime number, and that is the first in history to present the spiral placement of prime number this $i$ s the evidence for a spiral placement of prime number and the essential half-line values.

The unique relationship of space to the value 360 is presented here as a signature equation, based on the published divergence of empty space at 1:3 and 1:6

$$
\begin{aligned}
& \frac{360}{19}-X X=\frac{1}{19} \\
& 19-X X=\frac{1}{9.5} \\
& 9.5(1: 6) ; 19(1: 3)
\end{aligned}
$$

The gist of this manuscript is the clear mathematical proof that there is a mathematical disconnect between the linear mathematics as developed by man, and the primordial spiral planes and the half- line placement of the spiral /conal progression of mathematics. It is one thing to devise a linear prime number sieve and totally another to count prime numbers by their spirals and half - line placements. Thus mathematics could never come to term with the spiral planes 
of the universe of mathematics, except by indirectly placing the spiral sieve into a placement shown here by converting the linear sieve into a spiral sieve.

Keywords: Prime numbers distribution, -1 Vedic zero, Prime number 19; Unified Theorem; The final frontier in mathematics, Spiral calculus.

\section{Introduction}

Rather than aimlessly be discovering larger and larger prime numbers without understanding their genesis and framework, it is precise mathematics to understand Prime number mathematics before one does the mega hunting of prime numbers . That is what this Manuscript accomplishes without apology to current mathematicians. Cone-Metrics and its mathematical definition of the cone progression into empty space are at the very least fundamental to mathematics, and yet current mathematics does not have a definition of a half line of such a cone, which is essential, since the progression is curved. We have defined a 1:3 Cone and have defined the half-line in this manuscript

The following represents a clear concise proof of $-1,1: 3$ divergence and 1:6 convergence of the entire mathematics as is expressed in the several recently published papers of the author (Conundrum). The trivial obsession of current mathematics, linear prime number counting, and linear distribution is trivial because the functional expression of prime numbers is curved and spiral and the linear is simply an exercise in mathematics.

The differential between the base divergence and base convergence of the value 360 , as determined by reciprocal values $\left(\frac{1}{x}-\frac{1}{y}=\frac{1}{360}\right)$ is precisely the reciprocal of value 3 and $6(1: 3$ divergence and 1:6 convergence). This fact is mathematically immutable, if the reader understands the published paper in totality

The mathematics of the world's first and only continuous prime number sieve is based on the value 6 within the frame of 1:3 divergences, produced by Theo denOtter at Hope research. This is educational to current mathematics which touts non- continuous sieves of Prime numbers, which then need to be confirmed further. This continuous prime sieve has been tested by algorithm to over 10 million "directly connected" prime numbers. It is written by a farmer, and is fully tested.

The linear triviality of prime numbers and the Spiral Prime number placement sieve is presented here as an indirect precise sieve. The direct calculus for this determination is a logical mathematical impossibility with the current mathematics, a primarily linear mathematics, since prime numbers represent changing "spiral planes" which the author has demonstrated (as topologically shown in the authors published papers.).

The author cannot state this more simply and more succinctly that the continuum of all mathematics including numbers is divergent at 1:3 and convergent at 1:6, as confirmed by the published Pythagorean example at 1:3.

$$
\begin{gathered}
\frac{6 * 3}{\frac{6}{3}}=3^{2} \\
3^{2}+1=10 \\
3^{2}+10=19 \\
\left(3^{2}+10\right)-\left(3^{2}+1\right)=3^{2}
\end{gathered}
$$

It is vitally important to understand that the natural placement of prime numbers is a spiral configuration. The linear counting, sieve of prime numbers is unnatural and without function. Both these are clearly aligned to the value 6 .

The author has recently published several manuscripts in mathematical research, delineating the key role of -1 in the mathematics in empty space. Now the author tackles the numeration of Prime numbers with relation to the linear half line and number Placement. Unfortunately the role of Current mathematics has not been historically very enlightening as they have failed to differentiate between the placements of numbers versus the numeration of numbers. Prime numbers are placed spirally as our other publications have asserted. Whilst the pure numerations, is as by the sieve, the number of prime numbers and their distribution by numbers is a complex matter, and the solution is on the blackboard of the manuscripts in this conundrum .

The author holds these truths to be self-evident that all space and numerations, Including the value of 360 are all a manifestation of -1 as described in the authors published work on the unified theorem and -1 and that Prime 19 is the absolute manifestation of $1: 3$ and is a very unusual number (see the publication on the composite of 19 and the Vedic zero).

The rationality of the value 2 is related directly to 10 and 9: as follows.

$$
(10+10) *(9+9)=360
$$

as in $2-(2-0.2)=0.2$ or $20-(20-2)=2$ 


$$
\begin{gathered}
\text { or } 2 *(2-0.2)=3.6 \text { and } 20 *(20-2)=360 . \\
(2: 360) \sim(2:-1) \\
{[1-(1-2)=2]} \\
{[1 *(1-2)=-1]}
\end{gathered}
$$

\subsection{The 360 value and its mathematical accommodation:}

The following is a minor caveat but yet a cardinal proof of the whole mathematics that 360 is a primordial value and not an arbitrary value is intended for "Diaspora" to the general mathematicians, not just the elite.Please see our publication on Prime 19 and its key role

$$
\begin{aligned}
& \frac{360}{19}-X X=\frac{1}{19} \\
& 19-X X=\frac{1}{9.5}
\end{aligned}
$$

The author has validated the major role of prime number 19 at -1 extensively and has proved the correct trigonometry that $19 \sim 3$ and $9.5 \sim 6$.

The following mathematical values for the base values and end values of 360 are used to demonstrate and prove the standing of 19 in mathematics, something current mathematics has ignored.

$$
\begin{gathered}
\frac{1}{x}-\frac{1}{y}=\frac{1}{z} \\
\text { Base value for } 360=\frac{1}{36}-\frac{1}{40}=\frac{1}{360} \\
\text { End value for } 360=\frac{1}{(360-1)}-\frac{1}{(359 * 360)}=\frac{1}{360}
\end{gathered}
$$

That is what this manuscript is all about, a discussion of a narrow chiasm of mathematics and it is a clear proof of 19. The end value for,

$$
\frac{1}{X}-\frac{1}{Y}=\frac{1}{1} \text { is zero }
$$

Just recently the author has published -1 theorem including the Unified Theorem The author has just published a major thesis on the Unified Theorem and the Vedic zero at -1 at the International journal of applied Mathematics research and several other manuscripts at other journals referenced below, and since the mathematics has been presented in a nonSpartan manner, he had selected his journal "quarry" very carefully, as many western journals specially in the west are xenophobic to advancement in mathematics. Now since the work is published by 7 manuscripts, the author is presenting a narrow chiasm to drain current mathematics chronic obsession about the prime numerations, the number of Prime numbers and their distribution. This is done to avoid xenophobia of a new mathematics.

This mathematical expose is for the purpose of Diaspora. This pure mathematics question is related to this unified theorem, and the -1 mathematics of $191: 3$ presented as mathematics of the Pythagoras theorem at $1: 3: \sqrt{9}, \sqrt{10}$, in his published manuscripts. Currently the author is completing the complete resolution of Prime number distribution /placement based on the same principle.

$$
\frac{1}{x}-\frac{1}{y}=\frac{1}{360}
$$

There are infinite values for $\mathrm{X}$ and $\mathrm{Y}$ with positive whole number values .They start at (in sequential order), at the logical base. They end at the logical end value what is the calculus of the logical "end value"? This is shown as:

$$
\begin{aligned}
& \frac{1}{359}-\frac{1}{(359 * 360)}=\frac{1}{360} \text { end value } \\
& \frac{1}{36}-\frac{1}{40}=\frac{1}{360} \text { base value } \\
& \frac{1}{40}-\frac{1}{45}=\frac{1}{360} \\
& \frac{1}{60}-\frac{1}{72}=\frac{1}{360} \\
& \frac{1}{72}-\frac{1}{90}=\frac{1}{360}
\end{aligned}
$$




$$
\begin{aligned}
& \frac{1}{90}-\frac{1}{120}=\frac{1}{360} \\
& \frac{1}{120}-\frac{1}{180}=\frac{1}{360} \\
& \frac{1}{144}-\frac{1}{240}=\frac{1}{360} \\
& \frac{1}{160}-\frac{1}{288}=\frac{1}{360} \\
& \frac{1}{168}-\frac{1}{315}=\frac{1}{360} \\
& \frac{1}{180}-\frac{1}{360}=\frac{1}{360} \text {....52values } \\
& \frac{1}{359}-\frac{1}{(359 * 360)}=\frac{1}{360} \\
& \frac{1}{359}-\frac{1}{129240}=\frac{1}{360} \text { end value }
\end{aligned}
$$

Now reverse the calculus and understand 19 for once starting at the base value

$$
\begin{gathered}
\frac{40+36}{40-36}=19 \\
\frac{45+40}{45-40}=17 \\
\frac{90+72}{90-72}=9 \\
\frac{120+180}{180-120}=5 \\
\frac{144+240}{240-144}=4 \\
\frac{288+160}{288-160}=3.5 \\
\frac{315+168}{315-168}=3.2857142857 \\
\frac{360+180}{360-180}=3 \ldots . .52 \text { values } \\
\text { End value is }\left(\frac{\mathbf{1}}{\mathbf{3 5 9}}\right)-\frac{\mathbf{1}}{(\mathbf{3 5 9} * \mathbf{3 6 0})}=\frac{\mathbf{1}}{\mathbf{3 6 0}}
\end{gathered}
$$

By reverse calculus again for the end value:

$$
\frac{359+129240}{129240-359}=1.00557103064 . . \text { end value }
$$

What is the direct mathematical relationship between the base valuelend values by reverse calculus? Base value:

End Value:

$$
\begin{gathered}
\text { Base Value: } \frac{1}{x}-\frac{1}{y}=\frac{1}{360} \\
\frac{1}{36}-\frac{1}{40}=\frac{1}{360} \\
\frac{36+40}{40-36}=19
\end{gathered}
$$

$$
\begin{gathered}
\frac{1}{359}-\frac{1}{(359 * 360)}=\frac{1}{360} \\
\frac{359+129240}{129240-359}=1.00557103064 \\
\frac{19}{1.00557103064}=18.89473682112(X X)
\end{gathered}
$$

Note: 


$$
\begin{aligned}
& \frac{360}{19}-X X=\frac{1}{19} \\
& 19-X X=\frac{1}{9.5}
\end{aligned}
$$

This is proof of the Prime 19 at 1/360 that current mathematics has missed. THIS above is a MAJOR FINDING IN MATHEMATICS.19 1:3 and 9.5 1:6. Please refer to referenced publication on 19 and 1:3 divergences

\section{World's only continuous prime number sieve at value 6, 1:3:}

This is the method of Theo denOtter, who developed this at Hope research, but has declined to publish it thus far. However this is a continuous method, the most accurate and has been tested by algorithm till 10 million numbers. Since it is a continuous sieve it is precise and the prime numbers do not need any confirmation. This is the unedited rational method of the sieve in original, as follows in non standard mathematics language:

Hope research continuous sieve:(Method of Theo Denotter, Hope research and the world's only ever continuous prime number sieve). This continuous prime number sieve has been proven by 10 million continuous prime numbers by logarithm. The following is the unedited method of Theo denotter.

\section{Known fact:}

Prime numbers are one plus or one minus a divisible number, but when studied further they are all placed before or after a whole number that is divisible by the number 6.

In the case of the numbers one less than the whole number, it always has a residue of 5/6 whereas the number greater than the whole number it is always $1 / 6$ greater than the whole number.

Therefore, there are two distinct sets of prime numbers

\section{Example:}

Negative prime numbers:

$$
\begin{gathered}
\left(\frac{5}{6}\right)=0.83333333333 \text { or }\left(\frac{5}{6}\right) \\
\left(\frac{11}{6}\right)=1.833333333333 \text { or } 1 \text { plus }\left(\frac{5}{6}\right)
\end{gathered}
$$

Positive prime numbers:

$$
\begin{aligned}
& \left(\frac{7}{6}\right)=1.16666666666 \text { or } 1 \text { plus }\left(\frac{1}{6}\right) \\
& \left(\frac{13}{6}\right)=2.16666666666 \text { or } 2 \text { plus }\left(\frac{1}{6}\right)
\end{aligned}
$$

Therefore the setting as follows allows us a method of calculating if they are a prime or not. From the outer edges of the tangent setting a multiple of 5 and 6 is used

$\begin{array}{ll} & -1 \\ 1 & 5 \\ 7 & 11 \\ 13 & 17 \\ 19 & 23 \\ 25 & 29\end{array}$

Note: $5 * 6=30+1 * 5=35$

Note:

$5 * 6=30+35=65$ so the next multiple of the prime number 5 will be the number 65 .

Also, $7 * 6=42+35=77$ so the next multiple of the prime number 7 will be the number 77 


$$
\begin{array}{rr}
43 & 47 \\
7+42=49 & 53
\end{array}
$$

Note:

$7 * 6=42+49=91 / 7=13$

$13 * 6=78, \quad 91-78=13$

Also $91+78=169$

Note:

$55-30=25$ but also $55-66=-11$ which is the prime number on the -1 column

$55+30=85$ and $55+66=121$ multiples of 5 and 11

As the program runs all numbers that are left are prime numbers and will be multiplied by 6 and the rhythm of that number will then move forward to eliminate any multiples of itself.

When given a large number it can be divided by 6 and is absent the residue of 5/6 or 1/6 it will never be a prime number.

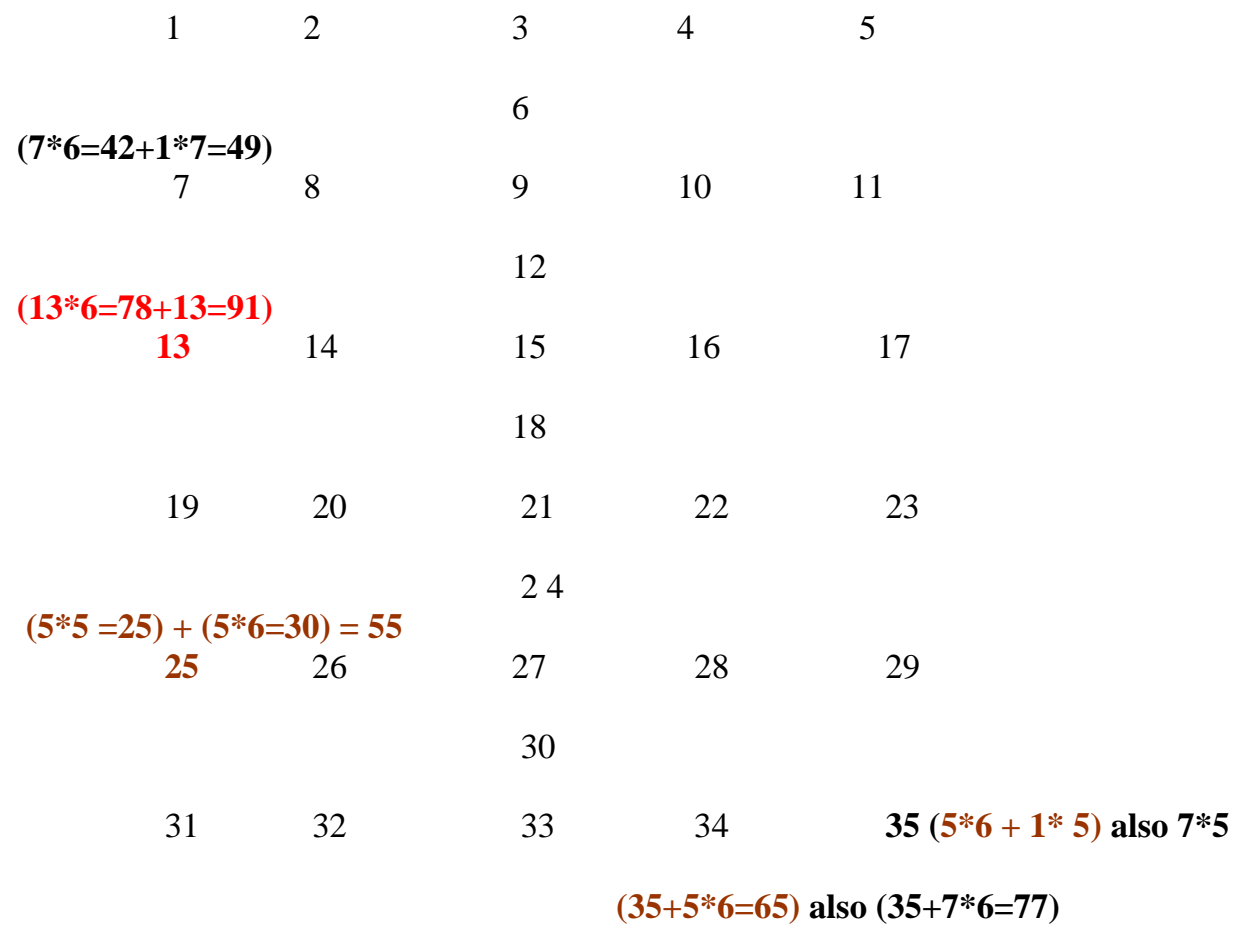

$(49+42=91)$ 
$(55+5 * 6=85)$

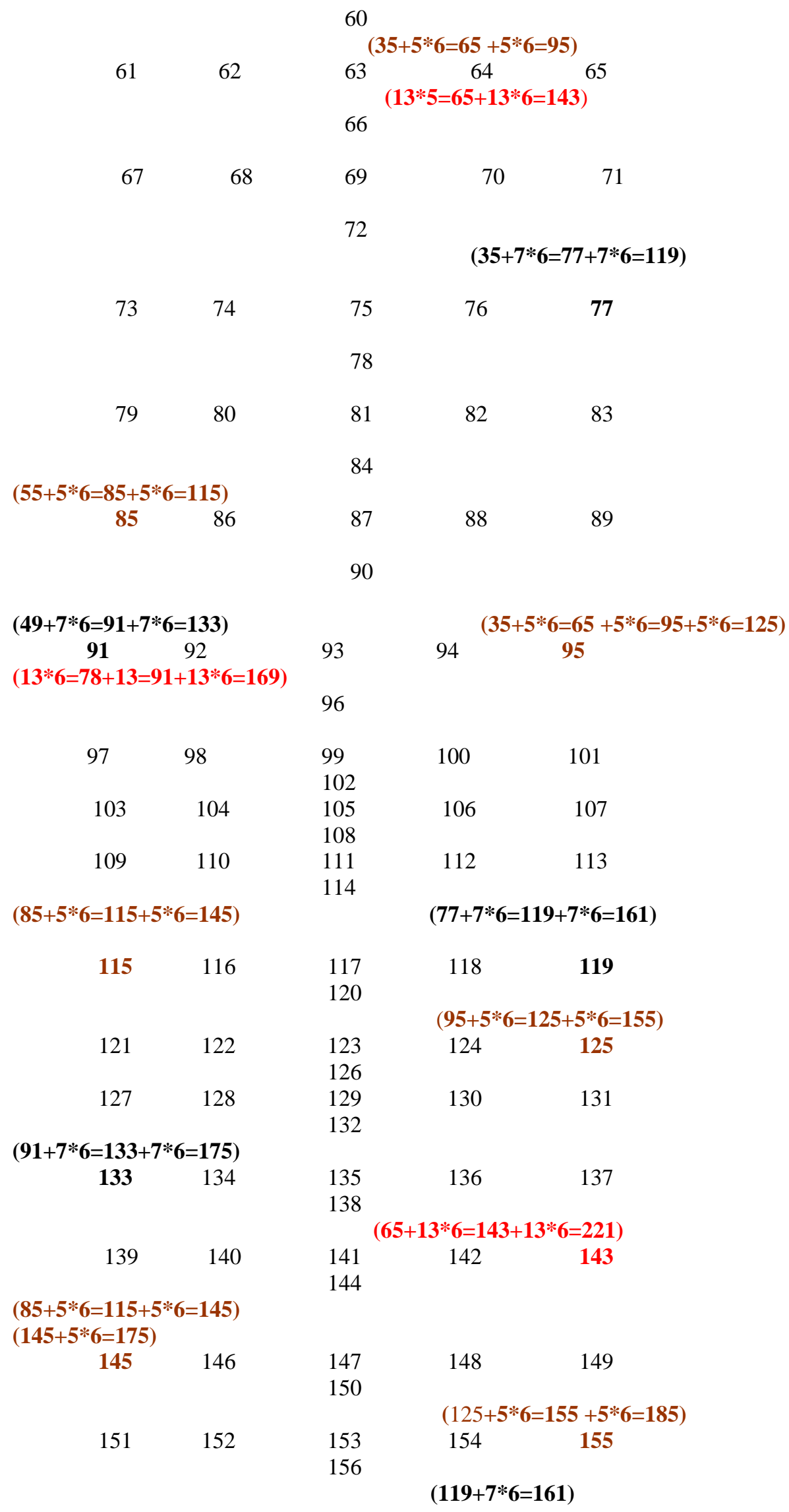




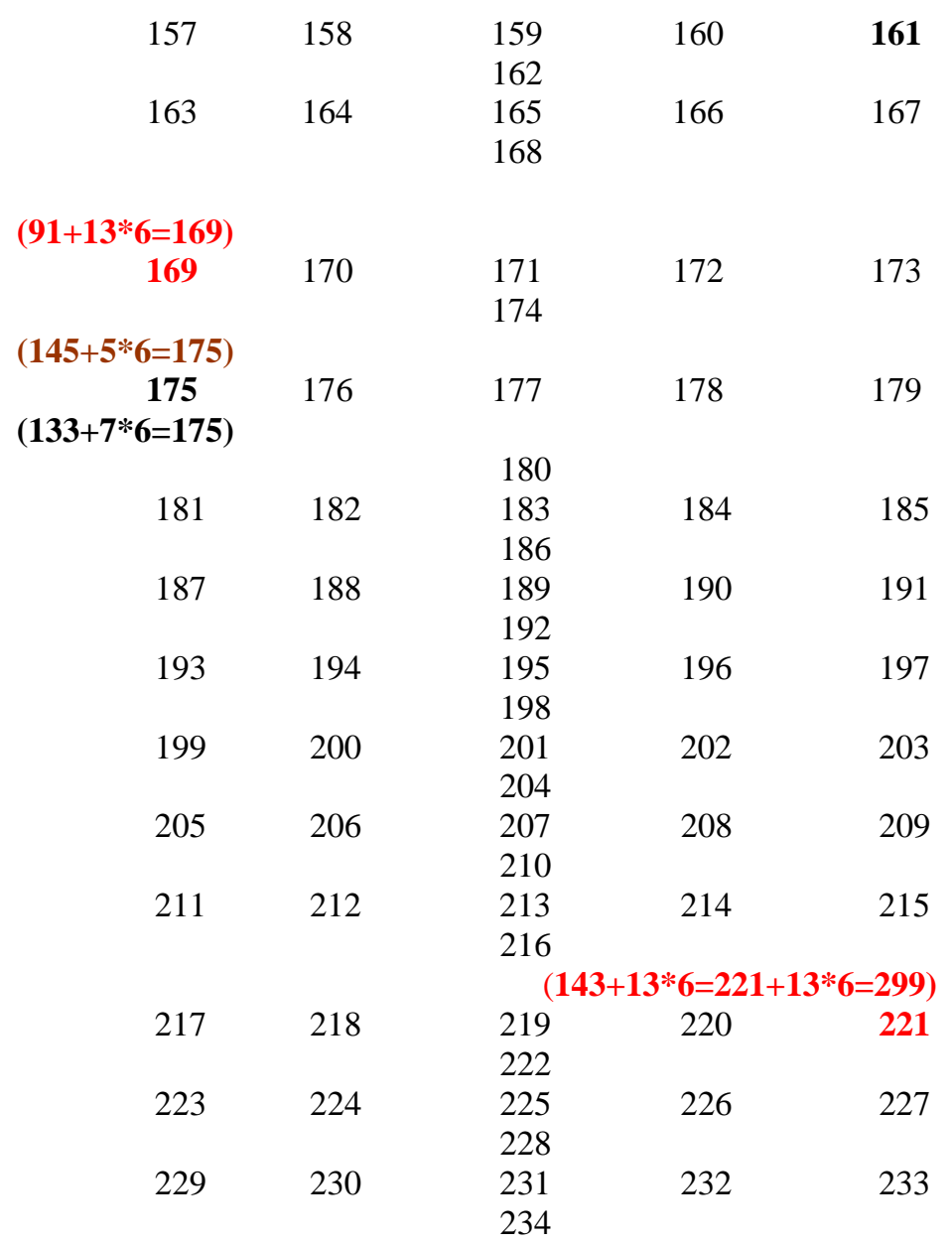

I have done only the 5, 7, and 13 prime numbers here so as to keep this less cluttered.

The basic prime numbers are already known but by an advancement of 6 gaps on each side the next possible prime is automatically put in its place.

Written by Theo den Otter / Hope Research

\section{Spiral placement of prime numbers}

An important fact current mathematics must be forced to understand is that Prime numbers have a spiral divergent placement in changing planes with reference to the half line and their values are very precise including a correct sieve such as shown above. This does not lend itself to linear mathematics. The author cannot make it more mathematically succinct.

These two cords are stable and mathematically mesh and divide perfectly into half-line values, divided into perfect whole divisions as segregated cords infinitely .Much of this is extensively published including the spiral placement. This basically shows a continuous mode of the two cords in a spiral weave. The arrangement is by the following examples for Cord a and Cord B.

Cord $A:(5 * 11)+(11 * 12)=11 * 17$.

Cord $B:(7 * 13)+(13 * 12)=(13 * 19)$

How to calculate half-line value of any number, here are three examples of infinite examples, but no mathematician should need more. Here it is for prime numbers $19,23,10140$

$$
\begin{gathered}
\frac{[(\mathbf{1 9} * 29)-(19 * 13)]}{19}=16 \\
\frac{[(\mathbf{2 3} * 31)-(23 * 17)]}{23}=14 \\
\frac{[(\mathbf{1 0 1 4 1} * 10151)-(10141 * 10111)]}{10141}=40
\end{gathered}
$$


Linear list of Prime numbers:

$5,7,11,13,17,19,23,29,31,37,41,43,47,53,59,61,67,71,73,79,81,87,97,101,103,107,109,113$

$127,131,137,139,149,151,157,163,167,, 173,179,181,191,193,197,199,211,223,227,229,233,239,241,251,257,263,269,2$ $71,277,281,283,293,307,311,313,317,331,337,347,349,353,359,369,373,379,383,389,397,401,409,419,431,433,443,44$ $9,457,461,463,467,479,487,491,499,503,509,521,523,541,547,557,563,569,571,577,587$

,593,599,601,607,613,617,619,631,641,,643,647

The two cords of prime number follow an ascension order as follows:

\section{Cord A:}

$5,11,17,23,31,41,47,59,67,73,83,97,103,109,127,137,149,157,167,179,191,197,211,227,233,241,257,269,277,283,307$, $313 ; 331 ; 347,353,369,379,389,401,419,433,449,461,467,487,499,509,523,(541,557)$, $569,577,593,601,613,619,641,647$

\section{Cord B:}

$7,13,19,29,37,43,53,61,61,71,79,89,102,107,113,131,139,151,163,173,181,193,199,223,229,239,251,263,271,281,293$, $311,317,337,349,359,373,383,397,409,431,443,457,463,479,491,503,521,547,563,571,587,599,607,617,631$

Linear list of Prime numbers and their Half-line values:

\begin{tabular}{|c|c|}
\hline 5 & 10 \\
\hline 7 & 11 \\
\hline 11 & 12 \\
\hline 13 & 12 \\
\hline 17 & 12 \\
\hline 19 & 16 \\
\hline 23 & 14 \\
\hline 29 & 18 \\
\hline 31 & 18 \\
\hline 37 & 14 \\
\hline 41 & 16 \\
\hline 43 & 16 \\
\hline 47 & 18 \\
\hline 53 & 18 \\
\hline 59 & 20 \\
\hline 61 & 18 \\
\hline 67 & 14 \\
\hline 71 & 18 \\
\hline 73 & 16 \\
\hline 79 & 22 \\
\hline 83 & 24 \\
\hline 89 & 22 \\
\hline 97 & 20 \\
\hline 101 & 18 \\
\hline 103 & 12 \\
\hline 107 & 12 \\
\hline 109 & 24 \\
\hline 127 & 28 \\
\hline 127 & 28 \\
\hline 137 & 20 \\
\hline
\end{tabular}


Spiral cords A, B, as shown above: * indicates base half value

\begin{tabular}{|c|c|c|c|}
\hline Cord A & Half-line & Cord B & Half-line \\
\hline 5 & 10 & 7 & 11 \\
\hline $11^{*}$ & 12 & 13 & 12 \\
\hline 17 & 12 & $19 *$ & 16 \\
\hline 23 & 14 & 29 & 14 \\
\hline 31 & 18 & 37 & 16 \\
\hline 41 & 16 & 43 & 18 \\
\hline 47 & 18 & 53 & 18 \\
\hline $59 *$ & 20 & 61 & 18 \\
\hline 67 & 14 & 71 & 18 \\
\hline 73 & 16 & 79 & 22 \\
\hline $83 *$ & 24 & $89 *$ & 18 \\
\hline 97 & 20 & 101 & 12 \\
\hline 103 & 12 & 107 & 24 \\
\hline 109 & 24 & 113 & 26 \\
\hline $127 *$ & 28 & $131^{*}$ & 20 \\
\hline 137 & 22 & 139 & \\
\hline & & & \\
\hline Examples: & & $=\left(137^{*} 149\right)$ & \\
\hline $\mathbf{1 3 7} 127$ & $+\left(137^{*} 22\right)$ & $=(19 * 29)$ & \\
\hline $\mathbf{1 9} 13$ & $+(19 * 16)$ & $\left.=141^{*} 10151\right)$ & \\
\hline $\mathbf{1 0 1 4} * 10111$ & $+\left(10141^{*} 40\right)$ & & \\
\hline
\end{tabular}

\subsection{File on mathematics Prime number spirals( Cone -metrics)}

Reference proof to the publishers of authors manuscript at IJAMR written by Vinoo Cameron MD of Hope research , Athens, Wisconsin , By the Inspiration of Jesus Christ, and with the friend ship of a great mathematician Belal Mohammed Batiha on the last day of April 2013.

Prime numbers are in bold, left column. The sequence has to be filled in after number 53, I do not have the inclination right now, and if the mathematicians cannot understand this final solution to prime spirals, they should believe in Albert Einstein and George Riemann forever.

$19-16=3$

$17+16=33$

$47+16=63$

23-14=9

$25+14=39$

$55+14=69$

29- $18=11$

$23+18=41$

$53+18=71$

31- $18=13$

$25+18=43$

$55+18=73$

$37-14=23$

$39+14=53$

$69+14=83$

$41-16=25$

$39+16=55$

$69+16=85$

$43-16=27$

$41+16=57$

$71+16=87$

47-18=29

$41+18=59$

$71+18=89$

53-18=35

$47+18=65$ 
$77+18=95$

$59-20=39$

$49+20=69$

$79+20=89$

61 $-18=43$

$55+18=73$

$85+18=103$

$67-14=53$

$69+14=83$

$99+14=113$

71 $-18=53$

$-65+18=83$

$-95+18=113$

$73-16=57$

$71+16=87$

$101+16=117$

79- $18=61$

$73+18==91$

$103+18=121$

$83-24=59$

$65+24=89$

$95+24=119$

$89-22=67$

$75+22=97$

$125+22=127$

101- $18=83$

$95+18=113$

$125+18=143$

$103-12=91$

$149+12=161$

157-18=139

$151+18=169(-2=167)$

$181+18=199(-2=197)$

173- $18=155$

$167+18=185^{* *}$

$197+18=215^{* *}$

191 $-18=173$

$185^{* *}+18=203(+50=253)$

$215^{* *}+18=233(+50=283$

193- $18=175$

$187+18=205(+60=265+30=295)$

$217+18=235(+30=265+30=295)$

271- $18=253$

$265+18=283$

$295+18=313$

For the final brief preliminary understanding ( Since this is a complex calculus), numbers with half- line values of 16 only produce values that are divisible by 3 or 5 , as this example.

Prime 229(is a spiral 16 number at -2 )

$229-16=213(71)$

$237+16=243(81)$

$257+16=272(91)$

The gap is 30 and $60(10,10)$

Prime 239(is not a spiral 16 number, it is a spiral 22 number at -14)

$239-16=223$

$237+16=253$ 


\section{The triviality of current prime number mathematics counting prime numbers and their linear distribution:}

Note: These are educational series

As shown in the continuous prime number sieve, the proportion 5 and 6 play a major role in the placement of prime numbers

This triviality of mathematics is pure mathematical logic and is irrelevant, and the author who has not completed this method fully, has no intention of spoon- feeding current mathematics on the counting of prime numbers .Mathematicians should see the clear hand writing on the wall. The triviality of Prime number counting and its linear sieve etc serves no purpose but is easily solvable, as we have shown in the continuous sieve in this manuscript and the following table. It is important that we understand that:

Basic:

$$
\begin{gathered}
4(-1)=3 \\
\left(4^{2} * 3^{2}\right)=144\left(12^{2}\right) \\
{\left[\frac{6^{2}}{5^{2}}\right] * 10^{2}=12^{2}=\left(4^{2} * 3^{2}\right)=144}
\end{gathered}
$$

The constant of 1.44 is a clear indicator that we have the basic coordinates to work on and that it is mathematically

\begin{tabular}{|c|c|c|c|c|}
\hline $\begin{array}{l}\text { Value at } \\
+6\end{array}$ & Prime number & $6-x=(5 * x)=$ & $\begin{array}{l}\text { Value at } * 5 \\
+5\end{array}$ & constant \\
\hline 6 & 5,7 & 1 & $\begin{array}{ll}5 & (6 * 6) /\end{array}$ & $(5 * 5)=1.44$ \\
\hline 12 & 11,13 & 2 & $10(12 * 6) /$ & $(10 * 5)=1.44$ \\
\hline 18 & 17,19 & 3 & $15(18 * 6) /$ & $(15 * 5)=1.44$ \\
\hline 24 & 23 & 4 & $20 \quad(24 * 6) /$ & $(20 * 5)=1.44$ \\
\hline 30 & 29,31 & 5 & $25 \quad(30 * 6) /$ & $(25 * 5)=1.44$ \\
\hline 36 & 37 & 6 & $30 \quad(36 * 6) /$ & $(30 * 5)=1.44$ \\
\hline 42 & 41,43 & 7 & $35 \quad(42 * 6) /$ & $(35 * 5)=1.44$ \\
\hline 48 & 47,49 & 8 & $40 \quad(48 * 6) /$ & $(40 * 5)=1.44$ \\
\hline 54 & 53 & 9 & $45 \quad(54 * 6) /$ & $(45 * 5)=1.44$ \\
\hline 60 & 59,61 & 10 & $50 \quad(60 * 6) /$ & $(50 * 5)=1.44$ \\
\hline 66 & 67 & 11 & $55 \quad(66 * 6) /$ & $(55 * 6)=1.44$ \\
\hline 72 & 71,73 & 12 & $60 \quad(72 * 6) /$ & $(60 * 5)=1.44$ \\
\hline 78 & 79 & 13 & $65 \quad(78 * 6) /$ & $(65 * 5)=1.44$ \\
\hline 84 & 83 & 14 & $70 \quad(84 * 6) /$ & $(70 * 5)=1.44$ \\
\hline 90 & 89 & 15 & $75 \quad(90 * 6) /$ & $(75 * 5)=1.44$ \\
\hline 96 & 97 & 16 & $80 \quad(96 * 6) /$ & $(80 * 5)=1.44$ \\
\hline 102 & 101,103 & 17 & $85 \quad(102 * 6) /$ & $(85 * 5)=1.44$ \\
\hline 108 & 107,109 & 18 & $90(108 * 6) /$ & $(90 * 5)=1.44$ \\
\hline 114 & 113 & 19 & $95(114 * 6) /$ & $(95 * 5)=1.44$ \\
\hline 120 & $119(121)$ & 20 & $100(120 * 6) /$ & $(100 * 5)=1.44$ \\
\hline 126 & 127 & 21 & $105(126 * 6) /$ & $(105 * 6)=1.44$ \\
\hline 132 & 131 & 22 & $110(132 * 6) /$ & $(110 * 5)=1.44$ \\
\hline 138 & 139,137 & 23 & $115(138 * 6) /$ & $(115 * 5)=144$ \\
\hline 144 & 143 & 24 & $120(144 * 6) /$ & $(120 * 5)=1.44$ \\
\hline 150 & 149,151 & 25 & $125(150 * 6) /$ & $(125 * 5)=1.44$ \\
\hline 156 & 157 & 26 & $130(156 * 6) /$ & $(130 * 5)=1.44$ \\
\hline 162 & 163 & 27 & $135(162 * 6) /$ & $(135 * 5)=1.44$ \\
\hline 168 & 167 & 28 & $140(168 * 6) /$ & $(140 * 5)=1.44$ \\
\hline
\end{tabular}
possible to understand the tabulations of Twin prime etc. The author has not yet finished the resolution, but it is obviously possible. 
Basic harmony of prime numbers at 1:3 divergence :Note that the two sides of the series is identical till the first pseudo prime number 49 , and then it changes and the series on the right then starts accommodating numbers divisible by 3 , but only after the first pseudo prime 49 . There is also mathematical slippage. There is a lot to understand about numbers theorem here. Basically the series is coordinated at 2 and 1.8 (20:18) which is the rational extension of 2, as an example 91(2)

77(1.8)

$$
\left(\frac{27.3}{15.16666666666}=2\right)
$$

$$
\begin{gathered}
\left(\frac{25.666666666}{12.8333333333}=1.8\right) \\
\frac{(+2 * 3)}{(+1.8) * 3.3333333333}=\frac{+0.33333333333 * 6}{+0.33333333333 * 6}=1
\end{gathered}
$$

\begin{tabular}{|c|c|c|c|}
\hline $1.6666666666666 * 3$ & $=5$ & $0.83333333333 * 6$ & $=5$ \\
\hline $2.1 * 3.33333333333$ & $=7$ & $1.16666666666 * 6$ & $=7$ \\
\hline $3.66666666666666 * 3$ & $=11$ & $1.83333333333 * 6$ & $=11$ \\
\hline $3.9 * 3.33333333333$ & $=13$ & $2.16666666666 * 6$ & $=13$ \\
\hline $5.6666666666666666 * 3$ & $=17$ & $2.83333333333 * 6$ & $=17$ \\
\hline $5.7 * 3.333333333333$ & $=19$ & $3.16666666666 * 6$ & $=19$ \\
\hline $7.666666666666666 * 3$ & $=23$ & $3.83333333333 * 6$ & $=23$ \\
\hline $7.5 * 3.333333333333$ & $=25$ & $4.16666666666 * 6$ & $=25$ \\
\hline $9.666666666666666 * 3$ & $=29$ & $4.83333333333 * 6$ & $=29$ \\
\hline $9.3 * 3.333333333333$ & $=31$ & $5.16666666666 * 6$ & $=31$ \\
\hline $11.666666666666666 * 3$ & $=35$ & $5.83333333333 * 6$ & $=35$ \\
\hline $11.1 * 3.33333333333$ & $=37$ & $6.16666666666 * 6$ & $=37$ \\
\hline $13.66666666666666 * 3$ & $=41$ & $6.83333333333 * 6$ & $=41$ \\
\hline $12.9 * 3.333333333333$ & $=43$ & $7.166666666666 * 6$ & $=43$ \\
\hline $15.66666666666666 * 3$ & $=47$ & $7.83333333333 * 6$ & $=47$ \\
\hline $14.7 * 3.333333333333$ & $=49$ & $8.16666666666 * 6$ & $=49$ \\
\hline $17.66666666666666 * 3$ & $=53$ & $8.5 * 6$ & $=51$ \\
\hline $16.5 * 3.333333333333$ & $=55$ & $8.83333333333 * 6$ & $=53$ \\
\hline $19.66666666666666 * 3$ & $=59$ & $9.16666666666 * 6$ & $=55$ \\
\hline $18.3 * 3.3333333333333$ & $=61$ & $9.5 * 6$ & $=57$ \\
\hline $21.66666666666666 * 3$ & $=65$ & $9.833333333333 * 6$ & $=59$ \\
\hline $20.1 * 3.333333333333$ & $=67$ & $10.16666666666 * 6$ & $=61$ \\
\hline $23.66666666666666 * 3$ & $=71$ & $10.5 * 6$ & $=63$ \\
\hline $21.9 * 3.333333333333$ & $=73$ & $10.83333333333 * 6$ & $=65$ \\
\hline $25.666666666666666 * 3$ & $=77$ & $11.166666666666 * 6$ & $=67$ \\
\hline $23.7 * 3.333333333333$ & $=79$ & $11.5 * 6$ & $=69$ \\
\hline $27.66666666666666 * 3$ & $=83$ & $11.833333333333 * 6$ & $=71$ \\
\hline $25.5 * 3.3333333333333$ & $=85$ & $12.166666666666 * 6$ & $=73$ \\
\hline $29.6666666666666666 * 3$ & $=89$ & $12.5 * 6$ & $=75$ \\
\hline $27.3 * 3.3333333333333$ & $=91$ & $12.833333333333 * 6$ & $=77$ \\
\hline 31.66666666666666666 & $=95$ & $13.5 * 6$ & $=81$ \\
\hline \multirow[t]{5}{*}{$29.1 * 3.3333333333333$} & $=97$ & $13.833333333333 * 6$ & $=83$ \\
\hline & & $14.666666666666 * 6$ & $=85$ \\
\hline & & $14.5 * 6$ & $=87$ \\
\hline & & $14.8333333333333 * 6$ & $=89$ \\
\hline & & $15.1666666666666 * 6$ & $=91$ \\
\hline
\end{tabular}




\section{Acknowledgements}

1. Jesus Christ who gave the author humility, wisdom, inspiration and standing amongst all the so called super mathematicians, by his special grace, truth.

2. Dr. Belal. Mohammed Batiha, PhD, a magnanimous mathematician and human being to the author.

3. Dr. Hong Ma PhD, the brave editor of Journal of American science, fostering creativity.

4. Editor, Science Asia, Mr. Hui- Win Lin. He is publishing a Diaspora to this manuscript

\section{References}

[1] Cameron.V.The unified Theorem at -1 (Vedic Zero), International Journal of Mathematics research, 2(2) (2013 221-251)

[2] Cameron.V, The disproof and fall of the Riemann's hypothesis by quadratic base: The correct variable distribution of prime numbers by the clear mathematics of the half-line values ("Chan function") of prime numbers, International Journal of Applied Mathematical Research, 2 (1) (2013) 103-110.

[3] Cameron V, den Otter T. Prime numbers 2012. Jam Sci 2012; 8(7):329-334]. (ISSN: 1545-1003), http://www.jofamericanscience.org.

[4] Cameron V, Prime number Coordinates and calculus J Am Sci, 2012; 8(10):9-10]. (ISSN: 1545-1003).http://www.jofamericanscience.org

[5] Cameron: Prime number19, Vedic Zero and the fall of western mathematics by theorem. International journal of applied mathematical research 2(1) (2013)111-115

[6] Cameron: The rational variability of all empty space by prime number: International journal of applied mathematical research, 2(2) (2013)157174 